\title{
Um Olhar sobre o Panorama Ambiental Nacional e Internacional dos Retardantes de Chama Bromados, em Particular os Éteres Difenílicos Polibromados
}

\author{
Ana Cláudia Gama ${ }^{1,2,3 *}$, Paula Viana ${ }^{1}$, \\ Bernardo Herold ${ }^{2}$, Damià Barceló ${ }^{3}$, João Bordado ${ }^{2}$
}

\begin{abstract}
P or forma a satisfazer as suas necessidades, muitos foram e são os compostos criados pelo Homem ao longo dos tempos. Destes compostos fazem parte os retardantes de cha$\mathrm{ma}$, mais concretamente os éteres difenílicos polibromados (PBDEs). Todavia, quando se tornaram claros os efeitos nocivos que os compostos criados têm sobre o ecossistema, começaram a desenvolver-se programas de monitorização com o intuito de se ter um conhecimento preciso da sua distribuição nos diversos compartimentos ambientais, assim como a sua concentração nesses mesmos compartimentos. Existem actualmente por todo o mundo inúmeros trabalhos de investigação sobre PBDEs. Este artigo visa dar uma ideia do panorama ambiental aquático nacional, não descurando, no entanto, o que se passa no resto do globo, a fim de realizar um estudo comparativo entre as concentrações de PBDEs detectadas em Portugal e noutros países.
\end{abstract}

\begin{abstract}
INTRODUÇÃo
O desenvolvimento tecnológico nos últimos anos fez disparar a utilização de polímeros sintéticos, equipamento electrónico e outros materiais de fácil ignição, em nossas casas e em praticamente todos os estabelecimentos comerciais, o que veio aumentar o risco de incêndios. De modo a minimizar, quer o risco de incêndios, quer o seu poder de propagação, recorre-se frequentemente à utilização de retardantes de chama [1].
\end{abstract}

Retardantes de chama são compostos, naturais ou sintéticos, que são adicionados a uma grande variedade de materiais, de que são exemplos os têxteis, os plásticos, os circuitos electrónicos e os materiais de construção, a fim de evitar ou, pelo menos, retardar a propagação do fogo. Actualmente, existem mais de 175 compostos químicos classificados como retardantes de chama, encontrando-se divididos em quatro grandes grupos: os inorgânicos (alumínio, antimónio e

Agência Portuguesa do Ambiente, Rua da Murgueira, Zambujal, Apartado 7585, 2720 Alfragide, Portugal

Departamento de Engenharia Química e Biológica, Instituto Superior Técnico, Av. Rovisco Pais

1049-001 Lisboa, Portugal

Departamento de Química Ambiental, IIQAB, CSIC

Jordi Girona 18-26, 08034 Barcelona, Espanha

*E-mail: claudia.gama@apambiente.pt estanho), os orgânicos halogenados (clorados e bromados), os organofosforados e os azotados [1, 2]. Todavia, em termos de mercado mundial têm importâncias bem distintas, conforme se pode constatar na Figura 1.

\section{Retardantes de chama bromados}

Os retardantes de chama orgânicos que têm maior relevo são os compostos bromados (BFRs), representando aproximadamente $20 \%$ do mercado mundial $[4,5]$. Estes compostos podem ser divididos em dois grupos: os denominados retardantes de chama aditivos, que não estabelecem qualquer tipo de ligação química, encontrando-se simplesmente misturados nos polímeros, de que são exemplo os éteres difenilícos polibromados (PBDEs), os bifenílicos polibromados (PBBs) e o hexabromociclododecano (HBCD) e os denominados reactivos, que formam ligações químicas com os materiais, fazendo parte deste último grupo o tetrabromobisfenol A (TBBPA) (Figura 2) [2, 6].

Os retardantes de chama bromados $\mathrm{R}-\mathrm{Br}$ têm a capacidade de retardar a propagação do fogo, porque, quando sujeitos a elevadas temperaturas, se decompõem, libertando radicais de bromo:

$\mathrm{R}-\mathrm{Br} \rightarrow \mathrm{R}^{\bullet}+\mathrm{Br}$

Estes reagem com o polímero $\mathrm{Pol}-\mathrm{H}$ onde se encontra o retardante:

$\mathrm{Pol}-\mathrm{H}+\mathrm{Br}^{*} \rightarrow \mathrm{Pol}^{\cdot}+\mathrm{H}-\mathrm{Br}$

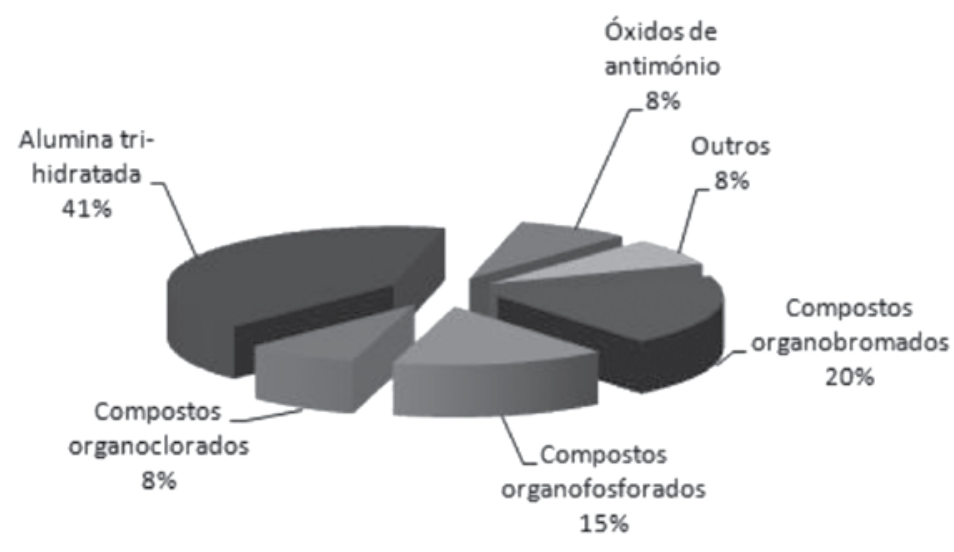

Figura 1 - Mercado mundial de retardantes de chama (toneladas $/ \mathrm{m}^{3}$ ) [3] 
O brometo de hidrogénio libertado in loco retarda os processos radicalares de decomposição endotérmica do polímero e da oxidação exotérmica dos líquidos e gases libertados. A sua acção retardante resulta da captação redutora dos radicais $R^{\cdot}$ que causam as reacções de propagação em cadeia e da ramificação das mesmas durante a combustão:

$$
\mathrm{R}^{\bullet}+\mathrm{H}-\mathrm{Br} \rightarrow \mathrm{R}-\mathrm{H}+\mathrm{Br}
$$

O brometo de hidrogénio é continuamente regenerado através da reacção da equação (2) e assim pode actuar de novo. Os radicais $R^{\cdot}$ podem ser quaisquer dos seguintes: $\mathrm{R}=\mathrm{Pol}^{\circ}$, Pol-O-O', Pol-O', H-O-O', HO', além de outros de que se sabe serem produzidos nos processos de decomposição e combustão do polímero. Reacções típicas são por exemplo as seguintes:

$$
\begin{aligned}
& \mathrm{Pol}-\mathrm{H}+\mathrm{O}_{2} \rightarrow \mathrm{Pol}^{\cdot}+\mathrm{H}-\mathrm{O}-\mathrm{O}^{\cdot} \\
& \mathrm{Pol}^{\cdot}+\mathrm{O}_{2} \rightarrow \text { Pol-O-O } \\
& \mathrm{H}-\mathrm{O}-\mathrm{O}{ }^{\circ}+\mathrm{Pol}-\mathrm{H} \rightarrow \mathrm{H}-\mathrm{O}-\mathrm{O}-\mathrm{Pol}+\mathrm{Pol}^{\bullet}(6) \\
& \mathrm{H}-\mathrm{O}-\mathrm{O}-\mathrm{Pol} \rightarrow \mathrm{Pol}-\mathrm{O}^{\circ}+\mathrm{H}-\mathrm{O}^{\circ} \\
& \mathrm{H}-\mathrm{O}^{\bullet}+\mathrm{Pol}-\mathrm{H} \rightarrow \mathrm{H}-\mathrm{O}-\mathrm{Pol}+\mathrm{Pol}^{\bullet}
\end{aligned}
$$

A equação (1) representa uma reacção de iniciação, (5) a (8) de propagação, sendo a (7) de bifurcação e, por isso, responsável pela aceleração do processo conducente à ignição [7].

\section{Éteres Difenílicos Polibromados}

De todos os retardantes de chama bromados, os primeiros a serem detectados no meio ambiente foram os éteres difenílicos polibromados, tendo-se verificado um aumento exponencial da sua concentração nas últimas duas décadas em diversas matrizes ambientais, nomeadamente no ar, nos sedimentos, nos peixes, mamíferos marinhos e até mesmo no Homem [2, 8-10].

O facto dos PBDEs terem propriedades semelhantes a alguns poluentes já bem conhecidos, nomeadamente os PCBs (bifenílicos policlorados), que ainda hoje, decorridas algumas décadas da sua proibição, constituem uma das classes de compostos mais detectados em matrizes ambientais, foi um dos factores que levou a comunidade científica a ter um interesse crescente no estudo dos PBDEs. Os PBDEs, assim como os PCBs, são persistentes, tóxicos, bioacumuláveis e possuem transporte a longa distância e, como tal, são considerados poluentes orgânicos prioritários, vulgarmente denominados por POPs. Para além destas propriedades, alguns PBDEs são também desreguladores endócrinos, interferindo no normal funcionamento da tiróide [11].

Existem 209 isómeros possíveis de PBDEs (a nomenclatura dos PBDEs baseia-se na mesma que a dos PCBs, IUPAC) divididos em 10 grupos de congéneres (mono até decabromado), no entanto, apenas um pequeno número é utilizado nas misturas comerciais [10]. Existem essencialmente três misturas comerciais: a PentaBDE, a OctaBDE e a DecaBDE e contêm, como o próprio nome indica, maioritariamente pentaBDEs, octaBDEs e decaBDE, respectivamente. Em 2001 a estimativa de mercado na União Europeia para o Penta foi de 150 toneladas, para o Octa de 610 to-

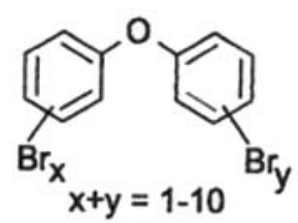

(a)<smiles>CC(C)(c1cc(Br)c(O)c(Br)c1)c1cc(Br)c(O)c(Br)c1</smiles>

(c)

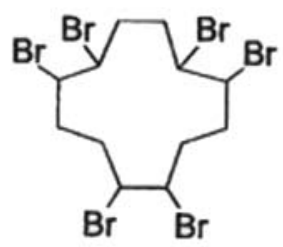

(b)

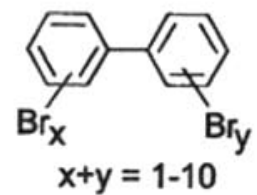

(d)
Figura 2 - Estrutura química dos: a) PBDEs, b) HBCD, c) TBBPA e d) PBBs neladas e de 7600 toneladas para o DecaBDE [5].

Actualmente, na União Europeia, quer a produção, quer a utilização da mistura PentaBDE comercial (composta essencialmente por BDE 47, BDE 99 e BDE 100) e da mistura OctaBDE comercial é proibida. No entanto, durante inúmeros anos foram utilizadas em grande escala, a primeira como aditivo de espuma de poliuretano, que por sua vez se destinava a mobiliário doméstico e à indústria automóvel e a segunda (95\%) como aditivo do poli(acrilonitrilo-butadieno-estireno) que era usado em computadores, telecomunicações, automóveis e equipamentos domésticos.

Dos três produtos comerciais, o DecaBDE é o único que actualmente pode ser utilizado e é constituído essencialmente por BDE 209 que é considerado de todos o menos móvel em termos ambientais e menos biodisponível devido ao seu elevado valor de $\mathrm{K}_{\text {ow }}$ (coeficiente octanol-água). Aliado à sua baixa solubilidade em água $\mathrm{e}$ reduzida volatilidade, o DecaBDE é utilizado em grande parte na indústria têxtil e de plásticos $[6,12]$.

\section{Fontes e Mecanismos de Liberta- Ção de PBDEs Para o MeIO} AMBiENTE

As emissões de PBDEs para o meio ambiente podem ocorrer em diversas fases: durante a própria produção dos PBDEs ou aquando da incorporação destes compostos nos produtos finais (plásticos, têxteis, etc...) através do material filtrado e do material rejeitado, enviados ambos para aterros; durante o ciclo de vida dos materiais aos quais foram adicionados, devido ao desgaste provocado pelo tempo e pelo uso. Existe também libertação nas águas municipais, o que explica os elevados valores encontrados nas lamas das ETARs. A emissão de PBDEs após o ciclo de vida útil dos materiais é susceptível de ocorrer por volatilização, lixiviação ou mesmo durante operações de reciclagem [12].

\section{Ocorrência de PBDEs no Meio Ambiente}

Devido à sua baixa volatilidade, baixa solubilidade e elevada afinidade por 
compostos de carbono, uma vez libertados para o ambiente, os PBDEs têm tendência a acumular-se nos sedimentos e solos. Por conseguinte, o ar e a água deverão apenas servir como meio de transporte. Foram encontrados PBDEs em diversos compartimentos ambientais, nomeadamente em lamas, em sedimentos, em peixes, em ostras, no ar, no cabelo humano e no leite materno em diferentes países por todo o mundo, mesmo em áreas remotas onde não são utilizados, o que indica que estes compostos possuem um elevado transporte aéreo [4].

Quanto é do conhecimento dos autores, em Portugal, apenas existem dados acerca dos teores de PBDEs no meio aquático, mais concretamente sedimentos e biota, Contudo, talvez seja interessante ver em termos mundiais o que se passa noutros compartimentos, nomeadamente, no ar, na água, etc...

\section{PBDEs no ar}

Existem poucos estudos acerca da concentração e distribuição de PBDEs no ar. Todavia, de um estudo realizado nos Estados Unidos foi possível concluir que os pentaBDEs e os hexaBDEs se encontram na atmosfera em locais relativamente perto das suas fontes, enquanto que os tetraBDEs são detectados mesmo em zonas longínquas, o que já era de prever, visto estes últimos terem pressões de vapor mais elevadas [13]. Tal como já era de prever, os PBDEs mais bromados, nomeadamente os octaBDEs, os nonaBDEs e o decaBDE, muito dificilmente se deslocam pelo ar, uma vez que têm pressões de vapor muito baixas. Assim sendo, a probabilidade de serem encontrados em concentrações detectáveis em áreas distantes das suas fontes é diminuta [14].

\section{PBDEs na água}

Os PBDEs têm baixa solubilidade em água, diminuindo à medida que aumenta o número de bromos. $O$ log $K_{\text {ow }}$ dos PBDEs, pelo contrário, aumenta à medida que aumenta o teor em bromo. Quer a solubilidade em água, quer o coeficiente de partição octanolágua, são bons indicadores do comportamento de um determinado com- posto em fase aquosa. Assim sendo, é de esperar que os compostos menos bromados, como por exemplo o BDE 47 e o BDE 99 sejam mais solúveis em água do que os mais bromados e, como tal, tenham uma maior mobilidade em água [15]. Na Europa, mais concretamente na zona costeira da Holanda (1999), foram registadas concentrações de BDE 47, BDE 99 e BDE 153 em amostras de água da ordem dos $1,0.5$ e $0.1 \mathrm{pg} \mathrm{L}^{-1}$, respectivamente. Na América do Norte, no Lago Ontário, também em 1999, foram detectadas concentrações de PBDEs em águas entre 4 e $13 \mathrm{pg} \mathrm{L}^{-1}$, sendo $90 \%$ destes PBDEs, BDE 47 e BDE 99. No Japão, em 1987-1988 verificou-se que não havia hexa, octa, ou decaBDE dissolvidos nas amostras de água (marinhas e estuarinas) recolhidas [15]. Todavia, num trabaIho desenvolvido na bacia do Ebro (Espanha), em 2006, detectaram-se concentrações elevadíssimas de BDE 209 em efluentes provenientes de indústrias têxteis [16].

\section{PBDEs nas lamas, no solo e nos sedimentos}

Tendo os PBDEs elevados coeficientes de partição sedimento-água, ao serem libertados para o meio ambiente têm tendência a acumular-se nos solos, nos sedimentos e nas lamas. Este compartimento ambiental toma assim especial importância, tornandose desta forma objecto de estudo de inúmeros grupos de investigação. É interessante verificar que os teores de PBDEs detectados nestas matrizes têm proporções semelhantes aos existentes nas misturas comerciais.

\section{Lamas}

$\mathrm{Na}$ Suécia, mais precisamente em Estocolmo, entre 1997-1998, foram realizados alguns estudos em lamas onde se verificou que a concentração de BDE 47, BDE 99 e BDE 100 nas lamas era 39-91 $\mathrm{ng} \mathrm{g}^{-1}$, 48-120 $\mathrm{ng} \mathrm{g}^{-1}$ e 11-28 $\mathrm{ng} \mathrm{g}^{-1}$ de peso seco (p.s.), respectivamente. Analisando estes dados constata-se que a razão das concentrações de alguns PBDEs $(B D E 47 /(B D E$ 99+BDE 100) $=0.61$ $0.66)$ detectadas nas lamas é semeIhante à razão da mistura comercial PentaBDE (BDE 47/(BDE 99+BDE
$100)=0.89$ [8]. Nos Estados Unidos da América foi desenvolvido um estudo idêntico tendo as conclusões sido semelhantes, ou seja, as proporções dos PBDEs detectadas nas lamas são concordantes com as existentes nas misturas comerciais [17].

Em Espanha foram analisados 14 PBDEs (BDE 7, BDE 15, BDE 17, BDE 28, BDE 47, BDE 49, BDE 66, BDE 85, BDE 99, BDE 100, BDE 119, BDE 153, BDE 154 e BDE 209) em lamas provenientes de seis estações de tratamentos e em todos os casos o congénere mais detectado foi o BDE 209 (93 a 99\%), o que indica a utilização da mistura DecaBDE. A concentração total de PBDEs variou entre 844 e $18100 \mathrm{ng} \mathrm{g}^{-1}$ p.s., sendo a concentração mais elevada detectada numa zona industrial com inúmeras indústrias têxteis [18].

Sedimentos e solos

Os sedimentos aquáticos e os solos terrestres são provavelmente o compartimento onde os PBDEs mais se depositam. A acumulação de PBDEs neste compartimento parece ser função, quer da distância à fonte, quer do teor de carbono orgânico total (TOC). De uma amostragem realizada em Portugal, entre 2002 e 2004, nas principais bacias hidrográficas, verificouse que todas as amostras de sedimentos estavam contaminadas com PBDEs, existindo contudo algumas discrepâncias no que diz respeito às suas concentrações. Dos 48 PBDEs analisados (mono a decaBDE) os mais detectados, à semelhança do que se verificou noutros países, foram o BDE 47, BDE 99, BDE 100 e BDE 209. Dos resultados obtidos constatou-se ainda que os sedimentos das bacias hidrográficas a norte estavam mais contaminados com PBDEs que os sedimentos das bacias hidrográficas a sul. A concentração total de PBDEs (mono a decaBDE) variou de 0.49 $\mathrm{ng} \mathrm{g}^{-1}$ a $126 \mathrm{ng} \mathrm{g}^{-1}$ p.s. o valor mais elevado foi detectado no sedimento de Ponte Nova de Barcelos, no rio Cávado, numa zona industrializada. Outros sedimentos que também têm concentrações apreciáveis de PBDEs são: Formariz (rio Ave) com $27 \mathrm{ng} \mathrm{g}^{-1}$ p.s., Ponte Moreira (rio Leça) $32 \mathrm{ng} \mathrm{g}^{-1}$ p.s., esteiro do Seixal (rio Tejo) $45 \mathrm{ng}$ 
$\mathrm{g}^{-1}$ p.s. e esteiro da Moita (rio Tejo) 44 ng $\mathrm{g}^{-1}$ p.s., todos eles localizados ou em zonas industrializadas ou junto a aglomerados urbanos. Verificou-se ainda que os níveis de contaminação, regra geral, aumentaram de 2002 para 2004, em especial no rio Cávado, no rio Leça e no rio Tejo [19].

Ainda em Portugal, numa amostragem de sedimentos realizada em 2004 no rio Trancão e nos seus principais afluentes (Ribeira de Loures e Ribeira da Póvoa), dos 47 PBDEs analisados foram detectados onze congéneres de PBDEs (BDE 47, BDE 49, BDE 77, BDE 99, BDE 100, BDE 196, BDE 197, BDE 203, BDE 206, BDE 207 e BDE 209). As concentrações totais de PBDEs variaram de 1.1 a $20 \mathrm{ng} \mathrm{g}^{-1}$ de peso seco e, tal como esperado, as concentrações mais baixas foram detectadas nos locais a montante das zonas mais industrializadas. Verificouse ainda que existia uma relação directa entre a concentração de PBDEs nos sedimentos e o teor de TOC [20]. Concentrações dentro da mesma ordem de grandeza foram detectadas em Espanha, mais concretamente na Catalunha, perto de Barcelona e Tarragona. Em sedimentos estuarinos e marinhos, a concentração total de PBDEs (mono até heptaBDEs) variou entre 0.86 e $2.49 \mathrm{ng} \mathrm{g}^{-1}$ de peso seco [21]. O BDE 209 foi detectado em todas as amostras variando a concentração de 2 a $132 \mathrm{ng} \mathrm{g}^{-1}$ de peso seco [22].

Um conjunto de grupos de investigação europeus desenvolveu um trabaIho em que realizaram amostragens de sedimentos nas embocaduras de 22 rios em vários países. De entre os compostos analisados constavam alguns PBDEs, nomeadamente o BDE 47, o BDE 99 e o BDE 209. Dos rios estudados os que apresentaram valores mais elevados de BDE 47 e BDE 99 nos sedimentos foram os rios Ingleses, nomeadamente o Rio Humber (BDE $47=6.2 \mathrm{ng} \mathrm{g}^{-1}$ p.s.; $\mathrm{BDE} 99=$ $7.0 \mathrm{ng} \mathrm{g}^{-1}$ p.s.) e o Rio Mersey (BDE 47 e BDE $99=7 \sim 2$ ng g $^{-1}$ p.s.). A concentração mais elevada de BDE 209 foi detectada também no Rio Mersey

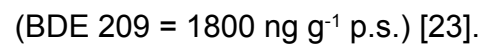

\section{PBDEs no biota}

Ao contrário do que se verifica nas lamas e sedimentos, no biota não se observa concordância entre a razão da concentração dos congéneres nas amostras e nas misturas comerciais. Este facto pode dever-se a dois factores: diferenças existentes na bioacumulação dos diversos congéneres e/ ou a mecanismos de degradação que porventura possam existir nos sistemas biológicos.

A persistência dos PBDEs em espécies aquáticas tem sido objecto de estudo, tendo especial interesse em países onde o consumo destas espécies é elevado, uma vez que os PBDEs, à semelhança de outros compostos orgânicos halogenados, podem ser transferidos ao longo da cadeia alimentar, originando biomagnificação. Em diversos estudos realizados no Mar Báltico com vários tipos de peixes comestíveis, mais concretamente arenque (Clupea harengus), espadilha (Sprattus sprattus) e salmão (Salmo salar) verificou-se sempre contaminação com PBDEs, sendo o congénere dominante o BDE 47 [24-26].

De um estudo realizado com Barbus $s p$. nos principais rios de Portugal verificou-se que, tal como nos sedimentos, existem diferenças nas concentrações de PBDEs nos peixes consoante a sua localização, sendo o BDE 47 o congénere mais detectado. A concentração de BDE 47 é maior no rio Douro e Mondego do que no Tejo, Sado e Guadiana. A concentração detectada no fígado dos peixes é superior à detectada no músculo, variando de 0.4 ng g-1 p.s. a 7.9 ng g $^{-1}$ p.s., no músculo e de 1.6 ng g $^{-1}$ p.s. a 30 ng g $^{-1}$ p.s. no fígado (Figura 3). Tal facto já era esperado visto o teor lipídico do fígado ser superior ao do músculo e, sendo os PBDEs hidrofóbicos e lipofílicos, se acumularem principalmente nos tecidos adiposos. Embora o BDE 99 e o BDE 100 tenham sido detectados em algumas amostras, a sua concentração é inferior à do BDE 47.

Estudos realizados na Suécia, mais concretamente no lago Bolman, situado numa zona cuja actividade agrícola e industrial têm vindo a sofrer um aumento considerável, verificou-se um aumento da concentração de BDE 47 nos peixes ao longo do tempo. Em 1968 a concentração de BDE 47 era inferior a 10 ng g $^{-1}$ de lípidos, em 1993 aumentou para $65 \mathrm{ng} \mathrm{g}^{-1}$ de lípidos e em 1997 passou a ser de $75 \mathrm{ng} \mathrm{g}^{-1}$ de lípidos [27]. Ainda na Suécia, em 1995, foram colhidas amostras de biota (Lúcio- Esox lucius) ao longo do Rio Viskan e verificou-se que a concentração total dos BDE 47, BDE 99 e BDE 100 variou entre 130 e $4600 \mathrm{ng} \mathrm{g}^{-1} \mathrm{de}$ lípidos, sendo o BDE 47, mais uma vez, o isómero predominante (50$-90 \%)$. As concentrações de PBDEs no biota aumentavam de montante para jusante, sendo os valores mais baixos detectados acima da localização das indústrias [28].

Num trabalho realizado em amostras de mexilhões (Mytilus galloprovincialis) colhidas em diversos locais da costa Portuguesa foram detectados alguns PBDEs, nomeadamente $\mathrm{O}$ BDE 47 (0.9-4.3 $\mathrm{ng} \mathrm{g}^{-1}$ de p.s.) (Figura 4) e o BDE 99 (0.3-1.6 ng g ${ }^{-1}$ p.s.) [29]. A concentração de PBDEs nos mexilhões é inferior à detectada nos peixes, tal não é de estranhar, visto os mexilhões pertencerem a um nível trófico inferior ao dos peixes analisados (Barbus sp), e estes compostos sofrerem biomagnificação ao longo da cadeia trófica. Outro dos factores que pode explicar o facto dos mexilhões terem concentrações mais baixas é o facto de viverem em zonas que regra geral estão menos contaminadas com este tipo de compostos, mais concretamente a zona costeira.

Valores da mesma ordem de grandeza foram obtidos em amostras de mexilhões (Mytilus edulis) colhidas na costa Holandesa onde foram detectados alguns PBDEs, nomeadamente o BDE 47 (0.9-4.3 ng g-1 de p.s.) e o BDE 99 (0.3-1.6 ng g ${ }^{-1}$ p.s.). O BDE 153 e o BDE 209, embora tenham sido analisados não foram detectados em nenhuma das amostras [30].

\section{Considerações Finais}

De tudo o que existe publicado sobre PBDEs uma ideia é clara, eles estão presentes em todos os compartimentos ambientais e em todos os pontos do globo e que, à semelhança do que aconteceu na década passada com os PCBs, devido à sua persistência mesmo depois de banidos, vão permanecer durante muito tempo no nosso ecossistema. 


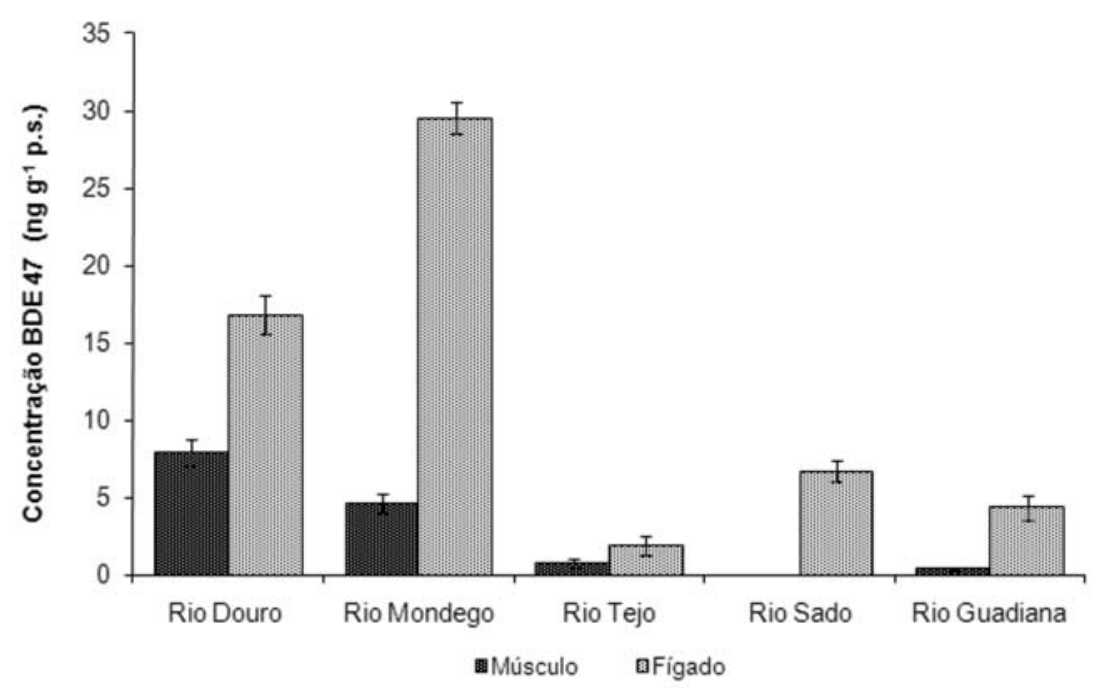

Figura 3 - Concentração de BDE 47 (ng g ${ }^{-1}$ p.s.) no músculo e no fígado de peixes (Barbus $s p$ ) colhidos no rio Douro, rio Mondego, rio Tejo, rio Sado e rio Guadiana em 2003

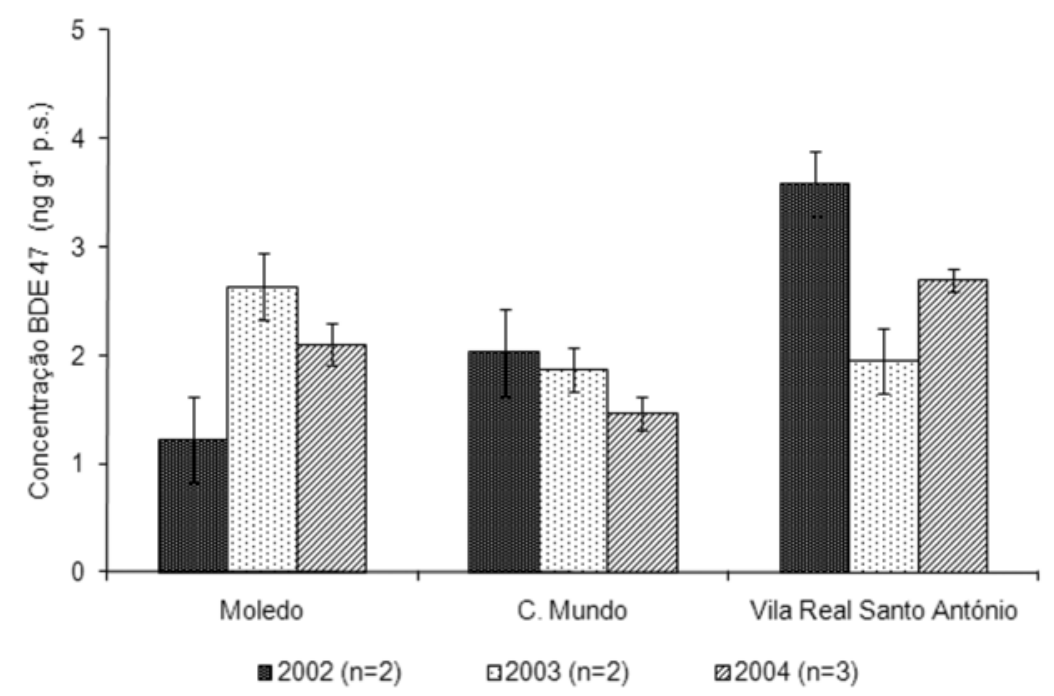

Figura 4 - Concentração de BDE 47 (ng g ${ }^{-1}$ p.s.) em mexilhões (Mytilus galloprovincialis) colhidos em diversos locais da costa Portuguesa em 2002, 2003 e 2004 [29] (n = número de amostras analisadas)

Os dados relativos à contaminação ambiental por PBDEs em Portugal são muito escassos, e tanto quanto é do conhecimento dos autores, existem apenas 4 trabalhos publicados [19, 20, 29, 31]. Todavia, os dados existentes já dão indicação dos níveis de contaminação do meio aquático em Portugal Continental, podendo-se concluir que as concentrações detectadas em Portugal, mais concretamente em sedimentos e biota, são da mesma ordem de grandeza das detectadas noutros pontos do globo, nomeadamente em Espanha, Holanda, Estados Unidos e Japão.

\section{Agradecimentos}

Os autores agradecem à Fundação para a Ciência e a Tecnologia, FCT (SFRH/ BD/14083/2003) o apoio financeiro.

\section{REFERÊNCIAS}

[1] M.Alaee, D.B. Sergeant, C.M Cannon, J.M. Luross, Proceedings of the biennial international conference on monitoring and measurement of the environment, Canada, 2000.

[2] M. Alaee, R.J. Wenning, Chemosphere 46 (2002) 579-582.

[3] CEFIC-EFRA, Web: http://www.ceficefra.com (European Flame Retardant Association, acedido em Setembro 2009).

[4] M.L. Hardy, Chemosphere 46 (2002) 717-728.

[5] BSEF, Web: http://www.bsef.com (Bromine Science and Environmental Forum, acedido em Setembro 2009).

[6] C. de Wit, Chemosphere 46 (2002) 583-624.

[7] J. Troitzsch, International Plastics
Flammability Handbook, Hanser Publishers, München, Wien, 1983.

[8] U. Sellström, A. Kierkegaard, T. Alsberg, P. Jonsson, C. Wahlberg, C. de Wit, Organohalogen Compounds $\mathbf{4 0}$ (1999) 383-386.

[9] N.G. Dodder, B. Standberg, R.A. Hites, Environmental Science and technology 36 (2002) 146-151.

[10] R.J. Wenning, Chemosphere 46 (2002) 79-796.

[11] J.B. Manchester-Neesvig, K. Valters, W.C Sonzogni, Environmental Science and technology 35 (2001) 1072-1077.

[12] J. Peltola, J. Ylä-Mononen, Finnish Environment Institute, Chemical Devision, Sweden (2000).

[13] B. Strandberg, N.G. Dodder, I. Basu, R.A. Hites, Environmental Science and technology 35 (2001) 1978-1083.

[14] S. Masten, B.L. Carson, Integrated Laboratory System (2001) 1-119.

[15] I. Watanabe, S. Sakai, Environmental International 29 (2003) 665-682.

[16] E. Eljarrat, A. de La Cal, D. Labandeira, D. Raldua, D. Barceló, $3^{\text {rd }}$ SWIFTWFD workshop em colaboração com Aquaterra, Barcelona, 2006.

[17] M.J. La Guardia, R.C. Hale, E. Harvey, The Third International Workshop on Brominated Flame Retardants. Toronto, Canada, 2004.

[18] B. Fabrellas, D. Larrazabal, M.A. Martinez, E. Eljarrat, D. Barcelo, Organohalogen Compounds 66 (2004) 3755-3760.

[19] A.C. Gama, P. Viana, D. Barcelò, J.C. Bordado, Organohalogen Compounds 68 (2006) 1767-1769.

[20] A.C. Gama, Sanatcumar P, P. Viana, D. Barcelò, J.C. Bordado, $3^{\text {rd }}$ SWIFTWFD workshop em colaboração com Aquaterra, Barcelona, 2006.

[21] E. Eljarrat, A. de la Cal, D. Barceló, Journal of chromatography A 1008 (2003) 181-192.

[22] E. Eljarrat, A. de la Cal, D. Raldua, C. Duran, D. Barceló Report SIME 97/6/1-E, Oslo and Paris Commissions (2004)

[24] P. Haglund, D.R. Zook, H. Buser, J. $\mathrm{Hu}$, Environmental Science and technology 31 (1997) 3281-3287.

[25] L. Asplund, M. Athanasiadou, A. Sjödin, Å. Bergman, H. Börjesson, Ambio 28 (1999) 67-76.

[26] T. Strandman, J. Koistinen, T. Vartiainen, Organohalogen Compounds 47 (2000) 61-64.

[27] A. Kierkegaard, U. Sellström, A. Bignert, M. Olsson, L. Asplund, B. Jans- 
son, C. de Wit, Organohalogen Compounds 40 (1999) 367-370.

[28] U. Sellström, A. Kierkegaard, C. de Wit, B. Jansson, Environmental Toxicology Chemistry. 17 (1998) 1065-1072.
[29] A.C. Gama, P. Sanatcumar, P. Viana, D. Barcelò, J.C. Bordado, Chemosphere 64 (2006) 306-311.

[30] J. de Boer, A. van der Horst, P.G. Wester, Organohalogen Compounds
47 (2000) 85-88.

[31] S. Lacorte, M. Guillamón, E. Martínez, P. Viana, D. Barceló, Environmental Science and technology 37 (2002) 892-898.

\section{Actualidades Científicas}

Nova Receita para Biodiesel Usa Cal

Investigadores reportaram um novo processo que utiliza cal hidratada para converter óleo de soja usado em biodiesel (Energy Fuels, DOI: 10.1021/ ef200555r). Este método alternativo apresenta potencial para o desenvolvimento de caminhos ambientalmente menos nocivos de produção de biocombustíveis.

A adopção de um sistema de cultivo agrícola dedicado somente à produção de combustíveis tem-se revelado controversa devido à sua contribuição para a redução relativa de superfície disponível para a produção de alimentos, para florestas e outros ecossistemas, assim como as suas implicações na utilização dos recursos aquíferos, principalmente em regiões reconhecidamente áridas. No entanto, no caso dos óleos vegetais usados ou residuais, a questão altera-se radicalmente, e muitos investigadores consideramnos como uma fonte desejável de matérias-primas para a produção de biodiesel, um substituto dos combustíveis fósseis obtidos a partir de gorduras vegetais ou animais.

Assim, Manuel Sánchez-Cantú da Autonomous Meritorious University of Puebla, no México, e seus colegas, aperceberam-se que a cal hidratada, ou hidróxido de cálcio, é facilmente disponível devido às suas múltiplas aplicações comerciais, nomeadamente na indústria alimentar, no tratamento de água, ou na refinação de petróleo. Por outro lado, a equipa sabia que outros investigadores tinham já verificado a possibilidade da aplicação do óxido de cálcio como catalisador eficiente do processo de transesterificação, que converte óleos vegetais em biodiesel através da reacção com metanol.

Deste modo, os investigadores testaram a viabilidade do processo em condições não laboratoriais (ou ditas de kitchen chemistry), recorrendo a reagentes comerciais obtidos em revendedores normais. Assim, usaram cal comprada em sacos de $25 \mathrm{~kg}$ num fornecedor de Perote, México, e óleo de soja obtido numa mercearia local, que foi utilizado para fritar batatas. Posteriormente, o processo de transesterificação do óleo usado foi promovido através da adição de metanol e de cal hidratada como catalisador. Após duas horas de reacção a uma temperatura de aproximadamente $60{ }^{\circ} \mathrm{C}$, obtiveram-se conversões próximas de $100 \%$. Adicionalmente, demonstrou-se que a cal podia ser reutilizada várias vezes, já que apenas na quarta utilização foi verificada uma degradação significativa do seu desempenho.

Pål Börjesson, um professor do Environmental and Energy Systems Studies, em Lund University, na Suécia, afirma que a utilização de cal comercial como catalisador é original. O material é não tóxico, vulgar e menos problemático do ponto de vista ambiental do que outros catalisadores como óxido de zinco e alumina. No entanto, o investigador realça que a substituição de combustíveis por produtos obtidos a partir de óleos alimentares usados é severamente limitada pela considerável diferença de escala entre a enorme procura de combustíveis comerciais e a relativa baixa disponibilidade de óleos residuais.

(Adaptado do artigo de 17/06/2011 de Naomi Lubick: New Recipe For Biodiesel Calls For Lime, Chemical \& Engineering News - http://pubs.acs.org/cen/news/89/ i25/8925scene4.html)

Paulo Brito (paulo@ipb.pt) Instituto Politécnico de Bragança

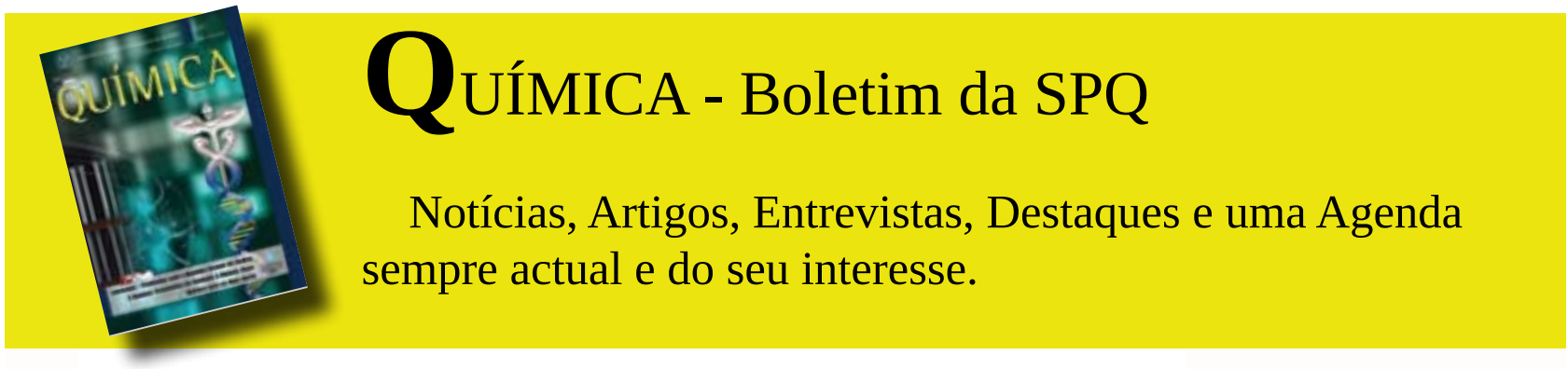

